\section{Gesellschaftliche Transformation - welche Rolle(n) für den Staat?}

\author{
Bei der saguf-Jahrestagung 2017 wurde \\ über die Rolle (n) des Staats für die \\ gesellschaftliche Transformation diskutiert: \\ Wie kann und soll der Staat auf gesellschaftliche \\ Transformationen einwirken?
}

saguf

schweizerische akademische gesellschaft für

umweltforschung und ökologie

societé académique suisse pour la recherche

sur l'environnement et l'écologie

swiss academic society for

environmental research and ecology
Basil Bornemann, Olivier Ejderyan, Michael Stauffacher, Patrick Wäger

Societal Transformation - What Role(s) for the State? | GAIA 27/1 (2018): 182-184

Keywords: societal transformation, state, sustainable development

W ie schon in den vergangenen Jahren (Zingerli und Küffer 2016, Moschitz und Moser 2017), hat die saguf auch 2017 die „gesellschaftliche Transformation“ zum Thema ihrer Jahrestagung gemacht. Diesmal richtete sich das Augenmerk auf den Staat. Um über die Rolle(n) des Staats bei der gesellschaftlichen Transformation zu

Kontakt Autoren: Dr. Basil Bornemann | Universität Basel | Departement Gesellschaftswissenschaften | Fachbereich Nachhaltigkeitsforschung | Basel | Schweiz |

E-Mail: basil.bornemann@unibas.ch

Dr. Olivier Ejderyan |

E-Mail: olivier.ejderyan@usys.ethz.ch

Prof. Dr. Michael Stauffacher |

E-Mail: michael.stauffacher@usys.ethz.ch

beide: ETH Zürich | Departement für Umweltsystemwissenschaften (D-USYS) | TdLab |

Zürich | Schweiz

Dr. Parick Wäger | Empa | Abteilung Technologie und Gesellschaft | St. Gallen | Schweiz | E-Mail: patrick.waeger@empa.ch

Kontakt saguf: saguf-Geschäftsstelle | Dr. Manuela Di Giulio | ETH Zentrum CHN | 8092 Zürich | Schweiz | E-Mail: saguf@env.ethz.ch | www.saguf.ch

(C) 2018 B. Bornemann et al.; licensee oekom verlag. This is an article distributed under the terms of the Creative Commons Attribution License (http://creativecommons.org/licenses/by/3.0), which permits unrestricted use, distribution, and reproduction in any medium, provided the original work is properly cited. diskutieren, fanden sich am 24. November 2017 im Volkshaus Zürich rund 60 Interessierte aus Wissenschaft, Politik und Zivilgesellschaft ein. Im Lauf der Veranstaltung wurde deutlich, dass das Thema nicht nur aktuell ist, sondern auch Raum für Kontroversen öffnet, die weiterer Diskussionen bedürfen.

\section{Der Staat verschwindet und wird wiederentdeckt}

In der umweltpolitischen Diskussion der 1970er und 1980er Jahre war der Staat allgegenwärtig. So dominierte die Vorstellung, dass die Bearbeitung gesellschaftlicher Umweltprobleme vor allem auf dem Wege staatlicher Steuerungsinterventionen gelingen könne. Im Zuge einer allgemeinen Ernüchterung über die tatsächlichen Möglichkeiten politischer Steuerung und des Aufkommens neuer Leitideen vom „Neoliberalismus“ bis zur „Nachhaltigkeit“ - rückte der Staat in den 1990er Jahren mehr und mehr aus dem Blickfeld der Diskussion über Möglichkeiten und Grenzen einer gezielten politischen Gestaltung von Gesellschaft. Die Diskussion konzentrierte sich stärker auf neue netzwerk- oder marktförmige Governance-Formen jenseits des Staats (Rhodes 1996). Seit einigen Jahren ist indes eine Wiederbelebung oder Wiederentdeckung des Staats zu beobach- ten. Ältere Thesen zu einem generellen Bedeutungsverlust des Staats sind neueren Erkenntnissen über einen Funktions- und Formwandel des Staats gewichen. Wenn auch nicht im Sinne einer hierarchischen „Command-and-Control-Politik“, könne der Staat doch als „Partner“ oder „Moderator“ im Rahmen komplex angelegter Governance-Arrangements auf gesellschaftliche Entwicklungen einwirken und diese im Sinne der Nachhaltigkeit gestalten (Duit et al. 2016, Meadowcroft 2007).

Im Zuge des Aufkommens verhaltenswissenschaftlicher Ansätze ergeben sich für einige Beobachter(innen) jüngst sogar neue Potenziale für einen aktiv steuernden Staat, der das Handeln seiner Bürger(innen) mittels sanften Schubsens (nudging) in die gewollte - auch nachhaltige - Richtung lenkt (Bornemann und Smeddinck 2016).

Abgesehen von diesen Veränderungsprozessen in Gesellschaft und Staat öffnet auch die internationale Politik neue Gelegenheitsfenster für eine Wiederentdeckung des Staats im Hinblick auf gesellschaftliche Transformationen. Erinnert sei hier an die Agenda 2030 für nachhaltige Entwicklung, die insbesondere auch die Rolle von Regierungen für die Koordination nationaler Transformationsprozesse thematisiert (Kläy et al. 2017). 


\section{saguf-Tagung zur Diskussion der Rollen des Staats}

Ziel der Jahrestagung war es, Interesse und Aufmerksamkeit für die aufkeimende Diskussion über die Rolle(n) des Staates in der gesellschaftlichen Transformation zu wecken und den Austausch zwischen Wissenschaft und Gesellschaft über das Thema zu befördern. Den Ausgangspunkt der Jahrestagung bildeten drei Fragen, die mit der nachhaltigkeitswissenschaftlichen Trias von System-, Orientierungs- und Transformationswissen korrespondieren:

1. Welche Rollen spielt der Staat schon jetzt in Bezug auf gesellschaftliche Transformation? (Systemwissen)

2. Welche „neuen“ Rollen des Staats wären mit Blick auf die Herausforderungen gesellschaftlicher Transformation in Richtung Nachhaltigkeit wünschenswert? (Orientierungswissen)

3. Wie ließen sich diese „neuen“ Rollen umsetzen und was wären die Folgen und Nebenfolgen davon? (Transformationswissen)

Diese Fragen wurden zunächst in Vorträgen von zwei Expert(inn)en aufgegriffen, die sich in unterschiedlichen Kontexten und aus unterschiedlichen Perspektiven mit dem Verhältnis von Staat und gesellschaftlicher Transformation beschäftigen.

Kora Kristof, Leiterin der Grundsatzabteilung beim deutschen Umweltbundesamt in Dessau und Autorin des Buchs Wege zum Wandel - Wie wir gesellschaftiche Veränderungen erfolgreicher gestalten können (Kristof 2010), fächerte in ihrem Eingangsbeitrag ein breites Spektrum möglicher Rollen des Staats für die gesellschaftliche Transformation auf. Ausgehend von einer Beschreibung zentraler Transformationsherausforderungen in einer zunehmend globalisierten und komplexen Welt identifizierte Kristof zunächst wichtige Hebel für gesellschaftliche Transformationen - angefangen bei der Gestaltung von Produktionssystemen und Märkten über die Ausrichtung öffentlicher Finanzen bis hin zu Entschleunigung und Zeitwohlstand. Darauf aufbauend erörterte sie schließlich Bedingungen für erfolgreiche Transformationsprozesse und diesbezügliche Rollen des Staats. Angesichts komplexer und dynamischer Systeme sowie erheblicher Widerstände und Konflikte könne der Staat etwa dazu beitragen, gemeinsame Visionen und Transformationsnarrative zu entwickeln, Allianzen zwischen transformationswilligen Akteuren aufzubauen, den Ausstieg aus nicht nachhaltigen Alltagspraktiken und Technologien zu fördern (Stichwort „Exnovation“), das Mainstreaming der Innovationen nachhaltiger $\mathrm{Ni}$ schenakteure zu stärken, gesellschaftliche Trends und Transformationsprozesse (wie die Digitalisierung) frühzeitig zu identifizieren und zu nutzen, die Fähigkeit aller gesellschaftlicher Akteure zur Mitgestaltung von gesellschaftlichen Wandelprozessen zu fördern oder selbst Debatten über gesellschaftliche Vorstellungen (zum Beispiel Wachstumsparadigma) anzustoßen, die einer nachhaltigen Entwicklung entgegenstehen.

Hans Widmer (alias P.M.), Experte für alternative Wohnformen und (Mit-)Autor oder -Herausgeber von Büchern wie Bolo' bolo (2015), Nach Hause kommen (Verein Neustart Schweiz 2016) und Die Andere Stadt (2017) vertrat in seinem Beitrag eine eher staatsskeptische (aber, wie er betonte, nicht libertäre, sondern anarchische) Position. Widmers Ausgangspunkt ist eine gegenüber Kristofs „Transformationsherausforderungen“ zugespitzte Lesart einer dreifachen - ökologischen, ökonomischen und psycho-sozialen - Krise der Gesellschaft. Diese mehrfache Krise würde nicht zuletzt auch insofern durch den Staat selbst erzeugt und verstetigt, als sich dieser im Sinne eines „Auffangstaats“ um die Bereitstellung systemrelevanter Funktionen kümmern würde (Stichwort „Rettung systemrelevanter Banken“). Der Ausweg aus dieser Krise bestünde in einer radikalen Transformation „unserer imperialen Lebensweise“. Diesen Ausweg skizziert Widmer vor allem in Form einer Vision für neue Wohnund Lebensformen. Zentrale Orte einer neuen Lebensweise, die „komfortabel, genussreich und vielfältig“ ist und ,alle glücklich macht“, seien Nachbarschaften und auf einer höheren Aggregationsebene Quartiere. In Widmers Vision würde der Staat vom „Auffangstaat“ zum „Ermöglichungs- und Unterstützungsstaat“. An- statt zu regulieren, würde der Staat Lebensweisen ermöglichen, „die es den Menschen erlauben, sich selbst zu regulieren“. Im Sinne des Subsidiaritätsprinzips würde er nur jene Leistungen erbringen, die nicht in der Nachbarschaft oder Quartieren erbracht werden können (beispielsweise Sicherheit, Gesundheit). Insgesamt gäbe es in dieser neuen Lebensweise von allem weniger - auch weniger Staat. Das Weniger an Staat würde aber durch ein Mehr an politischem Gemeinwesen („Commons“) kompensiert.

Die anschließende Diskussion, die von Claudia Vogt (sanu future learning ag, Biel) geleitet wurde, konzentrierte sich auf das Spannungsverhältnis gegenwärtiger und wünschbarer Rollen des Staats. Thematisiert und zum Teil kontrovers diskutiert wurden dabei vor allem die wechselseitigen Rollenerwartungen im Verhältnis von Staat und Bürger(inne)n und deren Veränderungen im Lauf der Zeit, Möglichkeiten, Notwendigkeiten und Grenzen legitimen staatlichen Handelns gegenüber der Gesellschaft sowie das Verhältnis von Staat und Demokratie.

\section{Ergebnisse: System-, Orientierungs- und Transformationswissen}

In Bezug auf die Ausgangsfragen haben Vorträge und Diskussion drei Bündel von Erkenntnissen zu den Rollen des Staats für gesellschaftliche Transformationen hervorgebracht.

In systemischer Perspektive hat die Veranstaltung erneut den Blick dafür geschärft, dass der Staat kein monolithischer Block ist. Vielmehr ist er ein komplexes und in sich differenziertes organisiertes Netzwerk von Akteuren, die in verschiedenen $\mathrm{Zu}$ sammenhängen unterschiedliche Rollen wahrnehmen können oder müssen. Der Staat ist der Ort, an dem konfligierende gesellschaftliche Werte, Ideen und Interessen institutionalisiert sind. Auch die Vorstellung, dass der Staat oder staatliche Akteure der Gesellschaft gegenüberstehen, ist demnach kaum zutreffend. Vielmehr ist der Staat mehr denn je „governancialisiert“, das heißt in Netzwerke eingebunden, in denen unterschiedliche staatliche, gesellschaftliche und wirtschaftliche Akteure kollektives Handeln zusammen gestalten. 
Infolgedessen sind die Rollen des Staats kaum isoliert zu betrachten, sondern müssen im Kontext komplexerer GovernanceArrangements gedacht werden. Durch ihre mannigfaltige Einbindung unterliegen staatliche Akteure in besonderem Maß institutionell, gesellschaftlich, kulturell und ökonomisch geprägten Rollenerwartungen. Sie sind qua Regeln und Konventionen zur Wahrnehmung bestimmter Rollen vative gesellschaftliche Nischen (Kristof) oder Nachbarschaften (Widmer) ermöglichen und schützen. Dafür müsse der Staat seine Bürger(inne)n ernst nehmen und stärken, anstatt sie steuern zu wollen. Das bedeute auch, dass diese auf der Ebene des Verstands und nicht auf der Ebene von Gefühlen angesprochen würden - ein bemerkenswerter Kontrapunkt zur gegenwärtigen Diskussion über nudging.

\section{Die Rollen des Staats müssen im Kontext komplexerer Governance-Arrangements gedacht werden.}

verpflichtet oder werden durch ökonomischen und politischen Druck in bestimmte Rollen gedrängt. Allerdings verfügen sie auch über Spielraum in der Interpretation und konkreten Ausgestaltung ihrer Rollen - und sie können Rollen jenseits etablierter Vorstellungen entwickeln und wahrnehmen.

Im Lauf der Veranstaltung wurden zahlreiche wünschbare Rollen des Staats thematisiert, die als orientierende Grundlage für das weitere Nachdenken über und die Gestaltung von Rollenmodellen dienen können. So wurde der Staat als „Begrenzer“ von gesellschaftlichen oder ökonomischen Entwicklungsprozessen gesehen, etwa im Hinblick auf die Einhaltung von planetary boundaries sowie als „Bewahrer" wesentlicher gesellschaftlicher und natürlicher Ressourcen. Überdies wurde die Rolle des Staats als „Regulierer“ von gesellschaftlichen und ökonomischen Machtverhältnissen thematisiert oder als aktiver „Gestalter“ gesellschaftlicher und ökonomischer Entwicklungen - im Sinne eines „Innovators“ und/ oder „Exnovators“. Dabei zeichneten sich in Bezug auf bestimmte Rollenmodelle durchaus kontroverse Positionen ab. Während einige Stimmen etwa in die Richtung einer aktiven Rolle des Staats argumentierten, der sich auch als Gestalter von gesellschaftlichen Werten betätigt, sprachen sich andere diesbezüglich eher für staatliche Zurückhaltung aus. Als zentral und über unterschiedliche Positionen hinweg anschlussfähig erscheint indes die Rolle des Staats als „Ermöglicher“. So soll er inno-
In transformationsorientierter Hinsicht bestand einerseits Konsens dahingehend, dass eine Veränderung der Rollen des Staats oder seines Rollenprofils (also der Zusammensetzung und Gewichtung unterschiedlicher Rollen) erforderlich sei. Mit Blick auf die gesellschaftliche, ökonomische und politische Einbettung des Staats wurde andererseits deutlich, dass es sich dabei um ein besonders schwieriges Unterfangen handelt. Schließlich geht es nicht nur darum, neue Rollenmodelle für den Staat zu etablieren, sondern auch alte Rollenverständnisse abzulegen. Diese Aufgabe einer Meta-Gestaltung der Rollen des Staats kann, so eine wesentliche Erkenntnis der Diskussion, nicht allein die Aufgabe des Staats sein, sondern wird selbst zu einer Aufgabe gesellschaftlicher Transformation. Gesellschaftliche Transformation kann also nicht allein auf einen Rollenwandel des Staats vertrauen, sondern muss aktiv an diesem mitwirken.

\section{Ein Blick nach vorn: Forschung und gesellschaftliche Debatte}

Insgesamt hat die Jahrestagung gezeigt, dass die Frage nach den Rollen des Staats für gesellschaftliche Transformationen nicht nur relevant und aktuell ist, sondern weiterer Vertiefungen bedarf - im Rahmen von Forschungsvorhaben einerseits und gesellschaftlichen Diskussionsprozessen andererseits.

Eine mögliche Forschungsperspektive besteht in der Rekonstruktion empirischer Rollenerwartungen und Rollenmodelle von
Staat und Gesellschaft in bestehenden oder zurückliegenden gesellschaftlichen Transformationsprozessen. Dadurch ließen sich Rollen staatlicher Akteure systematisieren und erfolgreiche - im Sinne transformationsfördernder - Rollenkonstellationen identifizieren, die als Bezugspunkt für die Gestaltung transformativer GovernanceArrangements dienen können. Zudem gilt es, die Frage nach den erwarteten, wünschbaren und möglichen Rollen des Staats auch zum Thema gesellschaftlicher Diskussionen zu machen. Angesichts der Verschränkung von Staat und Gesellschaft in komplexen Governance-Arrangements sollte es dabei allerdings weniger um die Rollen des Staats allein gehen, sondern vielmehr um eine Klärung von wechselseitigen Rollenerwartungen zwischen Staat und Gesellschaft. Im Sinne eines intensiveren Austauschs zwischen Wissenschaft und Gesellschaft über die Rollen des Staats für gesellschaftliche Transformationen wird die saguf das Thema auch zukünftig weiter bearbeiten.

\section{Literatur}

Bornemann, B., U. Smeddinck. 2016. Anstößiges Anstoßen? - Kritische Beobachtungen zur „Nudging"-Diskussion im deutschen Kontext. Zeitschrift für Parlamentsfragen 47/2: 437-459.

Duit, A., P. H. Feindt, J. Meadowcroft. 2016. Greening Leviathan: The rise of the environmental state? Environmental Politics 25/1: 1-23.

Kläy, A., C. Bader, B. Bornemann, V. Carabias, P. Wäger. 2017. Monitoring und Evaluation der Agenda 2030: Reflexionen zum ersten saguf-Gespräch. GAIA 26/3: 284-286.

Kristof, K. 2010. Wege zum Wandel: Wie wir gesellschaftliche Veränderungen erfolgreicher gestalten können. München: oekom.

Meadowcroft, J. 2007. Who is in charge here? Governance for sustainable development in a complex world. Journal of Environmental Policy and Planning 9/3: 299-314.

Moschitz, H., R. Moser. 2017. Urbane AgrikulturImpulse für eine nachhaltige Transformation von Stadt und Land. GAIA 26/1: 65-67.

P. M. 2015. Bolo'bolo. 7. Auflage. Zürich: Paranoia City.

Rhodes, R. A. W. 1996. The new governance: Governing without government. Political Studies 44: 652-667.

Verein Neustart Schweiz. 2016. Nach Hause kommen. Nachbarschaften als Commons. Baden: Ecoloc - Edition Volles Haus.

Widmer, H. (Hrsg.). 2017. Die Andere Stadt. Zürich: Paranoia City.

Zingerli, C., C. Kueffer. 2016. Eine große Transformation der Schweiz. Auf Bewährtes setzen und mit Neuem experimentieren. GAIA 25/1: 64-66. 